\title{
Community-Level Sanitation Coverage More Strongly Associated with Child Growth and Household Drinking Water Quality than Access to a Private Toilet in Rural Mali
}

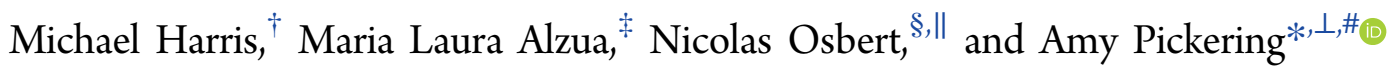 \\ ${ }^{\dagger}$ Emmett Interdisciplinary Program in Environment and Resources, Stanford University, Stanford, California 94305, United States \\ ¥CEDLAS-CONICET-Universidad Nacional de La Plata, La Plata, Buenos Aires 1900, Argentina \\ ${ }^{\S}$ UNICEF, New Delhi, 110 003, India \\ "UNICEF, Bamako, Mali \\ ${ }^{\perp}$ Civil and Environmental Engineering, Stanford University, Stanford, California 94305, United States \\ ${ }^{\#}$ Civil and Environmental Engineering, Tufts University, Medford, Massachusetts 02155, United States
}

Supporting Information

\begin{abstract}
Sanitation access can provide positive externalities; for example, safe disposal of feces by one household prevents disease transmission to households nearby. However, little empirical evidence exists to characterize the potential health benefits from sanitation externalities. This study investigated the effect of community sanitation coverage versus individual household sanitation access on child health and drinking water quality. Using a census of 121 villages in rural Mali, we analyzed the association of community latrine coverage (defined by a $200 \mathrm{~m}$ radius surrounding a household) and individual household latrine ownership with child growth and household stored water quality. Child height-for-age had a significant and positive linear relationship with community latrine coverage, while child weight-for-age and household water quality had nonlinear relationships that leveled off above $60 \%$ coverage $(p<0.01$; generalized additive models). Child growth and water quality were not associated with individual household latrine ownership. The relationship between community latrine coverage and child height was strongest among households without a latrine; for these households, each $10 \%$ increase in latrine coverage was associated with a $0.031(p$-value $=0.040)$ increase in height-for-age $z$-score. In this study, the level of sanitation access of surrounding households was more important than private latrine access for protecting water quality and child health.
\end{abstract}

\section{INTRODUCTION}

The post-2015 Sustainable Development Goal (SDG) for sanitation calls for universal access to adequate and equitable sanitation and an end to open defecation by $2030 .^{1}$ In 2015 , an estimated one billion people continued to practice open defecation of which approximately 165 million resided in rural sub-Saharan Africa. ${ }^{2}$ This lack of improved sanitation access contributes to a large global health burden, including mortality, diarrhea, trachoma, and helminth infections. ${ }^{3,4}$ Stunting, where a child's height-for-age is significantly (at least 2 standard deviations) below average, has also been attributed to inadequate sanitation through diarrheal illness and environmental enteropathy. ${ }^{5-8}$

The number of people practicing open defecation in subSaharan Africa increased from 181 to 229 million between 1990 and 2015 indicating a need for faster progress in increasing latrine access to meet the Sustainable Development Goals in sub-Saharan Africa. ${ }^{2}$ Additionally, while latrine installation has increased in many locations, usage of latrines does not necessarily follow. Two recent evaluations of large sanitation projects reported achieving household latrine coverage of $63 \%$ in Orissa, India, and 65\% in Koulikoro, Mali; subsequent usage of the latrines was reported at just $57 \%$ in India while it was nearly $100 \%$ in Mali. ${ }^{9,10}$ Environmental fecal pollution and community-health risks may persist when latrine coverage and usage are not universal.

Sanitation interventions strive to protect human health by safely containing fecal material and preventing its release into household and community environments. ${ }^{3}$ A household's sanitation access effects both the household members' health and the neighbors' health, yet community-level health effects have been difficult to quantify. ${ }^{11,12}$ Fecal pathogens from within one's own household are potentially less harmful than those from external sources, ${ }^{13}$ suggesting that community-level sanitation effects are important to understand. However,

Received: January 10, 2017

Revised: March 23, 2017

Accepted: May 17, 2017

Published: May 17, 2017 
exposure to fecal contamination within one's own household, regardless of the source, could be a health risk for young children with developing immune systems. ${ }^{14}$

A few studies have attempted to identify the effect of community-level sanitation coverage on child health in rural low-income settings. In Zimbabwe, a simple comparison of two communities, one with $67 \%$ latrine coverage and one with no latrines, found the community with latrines had $68 \%$ lower diarrhea prevalence. ${ }^{15}$ In Ecuador, improved sanitation coverage up to $\sim 30 \%$ led to significant improvements in child heightfor-age. ${ }^{16}$ In India, improved sanitation at the household-level was related to a $10 \%$ decrease in diarrhea prevalence, while reaching $100 \%$ improved sanitation coverage in the community was associated with an additional $37 \%$ reduction in diarrhea prevalence. ${ }^{17}$ Other studies have also shown that neighborhood or community open defecation rates have negative child health impacts. ${ }^{8,19,20}$ With the exception of the study in Ecuador, these analyses have relied on village-level estimates of latrine coverage or open defecation. A more accurate estimate for identifying external sanitation impacts may depend on a given distance threshold. ${ }^{18}$ In the Ecuador study, Fuller et al. focused on community access to pour-flush and improved pit latrines; ${ }^{16}$ evidence on externalities from upgrading communities from open defecation to basic sanitation would also be valuable.

A common hypothesis is that sanitation can improve child health by reducing drinking water contamination either in the household or at the source. ${ }^{3,14}$ Risk of fecal contamination of household stored drinking water in developing countries has been shown to increase where sanitation access is poor. ${ }^{14,25,26}$ Conversely, increases in the density of sanitation infrastructure $^{21,22}$ as well as the proximity to pit latrines have been associated with increased fecal contamination of source water. $^{23,24}$

In this study, we utilize a large spatial data set from rural Mali to analyze the effect of sanitation infrastructure and defecation behavior on child health outcomes, including diarrhea, weight, and height. We examine the relative importance of householdlevel sanitation versus community-level sanitation for protecting child health. We also explore the association between sanitation and household stored water quality as a potential pathway that sanitation can affect child health.

\section{METHODS}

Field Data Collection. The data for this study were collected during the census and baseline of a longitudinal evaluation of a community-led total sanitation intervention in Koulikoro, Mali. ${ }^{9}$ Prior to baseline household enrollment, a census of each study village was completed to locate all households with global position satellites (GPS) and record whether each household owned a latrine. Field enumerators were also identified and located with GPS community water sources within each study village. Households with children under the age of 10 identified during the census were enrolled into the sanitation impact evaluation study. In this paper, we classify households as "census" or "study" households to distinguish between the 1326 households with only census data available and the 4487 study households (with both census and household survey data available). Latrine presence and spatial location were identified for both census and study households; child health outcomes, reported open defecation, and water quality were measured in study households only. All data collection occurred during the dry season (March-June) in 2011.
For study households, field enumerators conducted in-home interviews with the female primary caretaker of the youngest child in the household. Participants provided oral consent prior to data collection. The study protocol was approved by the National University of La Plata (Buenos Aires, Argentina; protocol number 0001/2011 FC-UNLP), and Stanford University's (Stanford, CA, USA; protocol number 212090) human subjects and Institutional Review Boards.

Sanitation access was measured through interview questions as well as through observations of latrines by the field enumerators. Latrines in rural Mali are typically pit latrines with a solid mud platform. These latrines are classified as improved sanitation under the Joint Monitoring Program ${ }^{2,27}$ definitions if we consider the platform washable. In this manuscript, we will group this type of latrine construction with concrete slabs and refer to them both as basic sanitation. Open defecation practices were reported for the following household member categories: children less than 5 years old, boys and girls between 5 and 15 years old, men and women older than 15 years, and elderly.

Caretaker education and literacy was recorded as well as household assets (see the Supporting Information for details). Child health data was collected through self-report interview questions and anthropometric measurements. Health data was collected for 8133 study children. Caretakers were asked if each child under five had 3 or more loose or water stools in a $24 \mathrm{~h}$ period in the past 2 days. Pairs of trained anthropometrists measured the height and weight of all children under the age of five years. All weight and height measurements were taken in triplicate, and the median measurement was used for analysis. Children with height-for-age $z$-scores (HAZ) $<-2$ were classified as stunted. Children with weight-for-age $z$-scores (WAZ) $<-2$ were classified as underweight, and children with weight-for-height $(\mathrm{WHZ})<-2$ were classified as wasted.

Stored drinking water samples were collected from a randomly selected subset of seven study households in each village. Samples were processed for E. coli and total coliforms with the IDEXX Quanti-Tray/2000 method using Colilert-18 media to enumerate the most probable number (MPN) of bacteria per $100 \mathrm{~mL}$ of water sample. All drinking water sources in each village were identified and located with the help of local guides. Water quality samples were collected from the first five sources visited during the census and processed the same as stored water samples for E. coli and total coliforms.

Spatial Data Processing. The locations of all households, open defecation sites, and community water sources were imported into ArcGIS 10.3. A $200 \mathrm{~m}$ radius around each household was selected to calculate sanitation coverage; this radius was selected to capture sufficient variation in population density (an average of 33.8 households ( $S D=20.6$ ) were within a $200 \mathrm{~m}$ radius). Additionally, exposure to the environment within this radius was expected to be fairly common for both children and adults. ${ }^{28}$ Distances were measured in ArcGIS 10.3 using the Near Tool. Distances were calculated within the WGS 1984 UTM Projection, Zone 30. After identifying all households within a $200 \mathrm{~m}$ radius around each study household and water source, the household spatial data was then merged with household characteristics, water quality data, and child health outcomes for analysis.

Data Analysis. We assessed the relationship of sanitation to child health and water quality using two separate sanitation indicators: latrine coverage (an "infrastructure" indicator) and respondent-reported open defecation practices (a "behavior" 
indicator). One advantage of using latrine coverage over reported defecation behavior as an indicator in this study was that it was observed by enumerators and not subject to selfreport bias. We analyzed open defecation rates to account for whether or not household members actually used the latrines.

To determine community-level sanitation coverage for each study household, the number of households within the $200 \mathrm{~m}$ radius was used to calculate the percentage of households that owned a latrine. Thirty-two percent of study households reported having access to a neighbor's latrine; these households were not classified as having access to a latrine in our analysis. There were two reasons for this exclusion: (1) our census data was based on latrine ownership and therefore did not identify sharing; (2) sharing of sanitation facilities was associated with an increase in reported open defecation in our study population. To measure open defecation, we identified households where at least one household member older than 5 years of age reported open defecation as their main sanitation practice. Since we did not have reported open defecation practices for census-only households, we estimated the proportion of study households practicing open defecation in the $200 \mathrm{~m}$ radius as equivalent to the proportion of total households reporting open defecation. As $77 \%$ of total households are study households, this assumption seems reasonable. Household density was measured by counting the number of households within the $200 \mathrm{~m}$ radius.

We used multivariable regression to examine the relationship between community-level sanitation coverage, individual household sanitation access, and child health. Our infrastructure model included the presence of a latrine at the primary household and the proportion of households with latrines in a $200 \mathrm{~m}$ radius as independent variables. Our behavior model included self-reported open defecation practices in the primary household and the proportion of households reporting open defecation behavior within a $200 \mathrm{~m}$ radius as independent variables.

We analyzed continuous dependent variables (child growth $z$-scores) with linear regression and binary dependent variables (diarrhea prevalence and stunting, underweight, and wasting) with Poisson regression. We controlled for the number of households within $200 \mathrm{~m}$, household wealth proxied by asset ownership, whether the respondent could read or write, the child age in months, child gender, whether household drinking water was collected from an improved water source (i.e., protected shallow well, deep well, piped water), and villagelevel averages of wealth and literacy (averaged over data from study households only).

We also included an interaction term between household latrine ownership and latrine coverage within a $200 \mathrm{~m}$ radius. This method allows the relationship between household latrine ownership and child health to vary according to latrine coverage within $200 \mathrm{~m} .{ }^{17}$ Additionally, we considered the potential for a nonlinear relationship between community-level latrine coverage and child health. To first assess if a nonlinear relationship was present, we graphed localized polynomials to visualize the relationship between latrine coverage and weightfor-age, height-for-age, and weight-for-height $z$-scores. We then completed a regression analysis with a second-order term for $200 \mathrm{~m}$-radius latrine coverage allowing for potential diminishing or increasing returns to health as sanitation coverage approached $100 \%$. Regression analyses were also stratified by gender. Robust standard errors were calculated for each regression to account for clustering at the village-level. To interpret the statistical significance and magnitude of the associations between sanitation and child health outcomes for second-order regressions and linear regressions with interaction terms, marginal effects were calculated for $10 \%$ increases in community latrine coverage starting at $20 \%, 40 \%$, and $60 \%$ coverage (approximately corresponding to the quartile breaks of $20 \%, 43 \%$, and $62 \%$ for the sample population).

We also used generalized additive models to explore nonparametric relationships between community latrine coverage and child growth. The generalized additive models allowed for nonlinear relationships with each of the continuous covariates included in the regression models: latrine coverage, asset index, number of neighboring households, and village wealth and literacy averages.

To assess the relationship between community sanitation coverage, individual household sanitation access, and household stored water quality, we used linear multivariable regression with $E$. coli and total coliform concentrations as the dependent variables. We examined linear relationships for both the infrastructure and behavior models and second-order models for infrastructure only. We controlled for the number of households within $200 \mathrm{~m}$, whether stored water was treated (chlorine or boiling), whether the storage container was covered, household wealth as indicated by asset ownership, whether the drinking water was collected from an improved water source, and village-level average wealth and literacy. We also analyzed the relationship between stored water quality and latrine coverage with an interaction term between householdlatrine ownership and latrine coverage within $200 \mathrm{~m}$ along with a second-order latrine coverage term. We also computed marginal effects and generalized additive models for water quality outcomes. The source water quality analysis is detailed in the Supporting Information.

We used Stata v14 for the majority of the analyses. For generalized additive modeling, we used the package "gam" in $\mathrm{R}$ v3.3.2.

\section{RESULTS}

Household and Village Statistics. A total of 121 villages and 5813 total households were identified during the census and included in the analysis (64 households were dropped after spatial cleaning). Our analysis included 4487 study households and 1326 census-only households. A village consisted of 48 households on average [range of 27-134]. The mean latrine coverage within a village based on census data was $41.7 \%$ with a minimum of no latrines (11 villages) and a maximum of $100 \%$ coverage (1 village). Histograms showing household density and the percent latrine coverage surrounding study households within a $200 \mathrm{~m}$ radius are provided in Figure S1.

Study households had an average of 7.6 total members including 1.7 children under the age of 5 years. Ninety-three percent of households reported that children in the household practiced open defecation as their main defection location. Sixty-one percent of households reported that a nonchild household member practiced open defecation. Forty-two percent of households reported using a private latrine. Of households with a private latrine, 55\% shared the latrine with a neighbor. Eleven percent of households had access to a latrine with a concrete slab connected to a septic tank or a ventilated improved pit latrine. ${ }^{2}$ Regardless of construction type, $53 \%$ of latrines were observed to have a cover placed over the drop hole, and $97 \%$ of latrines observed showed signs of regular use. 
Children were on average 26.9 months old (range of newborn to 60 months). Thirty percent of children were stunted, and $28 \%$ were underweight. Using a 2-day recall period, self-reported diarrhea in a child under five years was prevalent in $20 \%$ of the population. Household characteristics, child health, and water quality statistics are provided in Table 1.

Table 1. Summary Statistics for Household Characteristics, Child Health Outcomes, and Water Quality

\begin{tabular}{|c|c|c|c|}
\hline variable & $N$ & mean & SD \\
\hline \multicolumn{4}{|c|}{ Household Characteristics } \\
\hline household has a latrine (\%) & 5813 & 41.6 & 49.3 \\
\hline latrine cover present (\%) & 2916 & 53.1 & 49.9 \\
\hline latrine has soap (\%) & 2912 & 2.92 & 16.8 \\
\hline latrine has flies (\%) & 2913 & 63.3 & 48.2 \\
\hline latrine appears regularly used (\%) & 2907 & 94.4 & 22.9 \\
\hline open defecation child ( $<5$ years) main $(\%)$ & 4033 & 92.6 & 26.2 \\
\hline open defecation individual ( $>5$ years) main (\%) & 4368 & 60.8 & 48.8 \\
\hline improved drinking water source (\%) & 4332 & 43.1 & 49.5 \\
\hline own mobile phone (\%) & 4486 & 40.6 & 49.1 \\
\hline literate $(\%)$ & 4212 & 31.2 & 46.3 \\
\hline latrine coverage, $200 \mathrm{~m}(\%)$ & 4487 & 43.4 & 28.2 \\
\hline $\begin{array}{l}\text { households (>5 years) open defecation, } \\
200 \mathrm{~m}(\%)\end{array}$ & 4487 & 65.9 & 25.7 \\
\hline \# people in household & 4487 & 7.61 & 3.94 \\
\hline \# households, $200 \mathrm{~m}$ & 4487 & 33.4 & 20.48 \\
\hline \multicolumn{4}{|c|}{ Child Health Outcomes } \\
\hline height-for-age, $z$-score & 6352 & -1.18 & 1.59 \\
\hline weight-for-age, $z$-score & 6364 & -1.27 & 1.42 \\
\hline weight-for-height, $z$-score & 6155 & -0.87 & 1.42 \\
\hline stunted & 6347 & 0.30 & 0.46 \\
\hline underweight & 6364 & 0.28 & 0.45 \\
\hline wasting & 6155 & 0.19 & 0.39 \\
\hline diarrhea, 2-day recall & 6817 & 0.20 & 0.40 \\
\hline \multicolumn{4}{|c|}{ Water Quality Outcomes } \\
\hline household stored water quality $E$. coli & 835 & 2.15 & 1.01 \\
\hline $\log \mathrm{MPN}$ per $100 \mathrm{~mL}$ & 835 & 3.22 & 0.39 \\
\hline source water quality & 395 & 2.28 & 1.38 \\
\hline $\log \mathrm{MPN}$ per $100 \mathrm{~mL}$ & 395 & 2.88 & 1.05 \\
\hline
\end{tabular}

In our study, household-level access to a latrine was associated with lower open defecation prevalence. Households which owned a private latrine were significantly less likely to have individuals of all age groups and genders report practicing open defecation, as compared to households that shared a neighbor's latrine. As compared to households with private latrines, individuals between 5 and 15 years old were 29\% ( $p<$ $0.001)$ more likely to report practicing open defecation when they had access to a neighbor's latrine and 5\% $(p<0.05)$ more likely when they owned a shared latrine. Children under the age of 5 years practiced open defecation $87 \%(\mathrm{SD}=34 \%)$ of the time in households with private latrines and 94\% ( SD = 25\%) of the time in households sharing a neighbor's latrine. Comparisons of open defecation behavior by age group and sanitation access type are provided in Tables S1 and S2.

Child Health. Child health and anthropometric data were collected in 3810 households with at least one child under the age of 5, for a total of 6894 children. First-order linear regressions indicated statistically significant relationships between higher community latrine coverage and improved height-for-age ( $p$-value $=0.024)$ and reduced prevalence of a child being underweight $(p$-value $=0.024)$ and a marginally statistically significant relationship with stunting ( $p$-value = 0.057) (Tables S3 and S4). Latrine ownership at the household level was significantly associated with lower diarrhea $(p$-value $=$ 0.047). The association between diarrhea and household latrine ownership was no longer significant when an interaction between latrine ownership and latrine coverage was included (Tables S3 and S4). The percentage of households practicing open defecation in a $200 \mathrm{~m}$ radius had a statistically significant association with increased prevalence of underweight ( $p$-value $=$ $0.021)$ and marginally significant association with decreased weight-for-height $z$-scores $(p$-value $=0.053)$. Open defecation at the household level was not significantly associated with any child health outcomes (Table S5).

The localized polynomial for height-for-age and stunting indicated a linear relationship with latrine coverage, while localized polynomials of weight-for-age, weight-for-height, underweight, and wasting suggested nonlinear relationships with community latrine coverage in a $200 \mathrm{~m}$ radius (Figure S2).

Table 2. Marginal Effects of Household Latrine Ownership and Community-Level Latrine Coverage for Each Health Outcome and Stored Water Quality Measure ${ }^{a}$

\begin{tabular}{|c|c|c|c|c|c|c|c|c|c|}
\hline \multirow[b]{3}{*}{ outcome } & \multicolumn{3}{|c|}{$\%$ latrine coverage $=20 \%$} & \multicolumn{3}{|c|}{$\%$ latrine coverage $=40 \%$} & \multicolumn{3}{|c|}{$\%$ latrine coverage $=60 \%$} \\
\hline & \multirow[b]{2}{*}{$\begin{array}{l}\text { household } \\
\text { latrine }\end{array}$} & \multicolumn{2}{|c|}{$\%$ latrine coverage } & \multirow[b]{2}{*}{$\begin{array}{l}\text { household } \\
\text { latrine }\end{array}$} & \multicolumn{2}{|c|}{$\%$ latrine coverage } & \multirow[b]{2}{*}{$\begin{array}{l}\text { household } \\
\text { latrine }\end{array}$} & \multicolumn{2}{|c|}{$\%$ latrine coverage } \\
\hline & & no latrine & owns latrine & & no latrine & owns latrine & & no latrine & $\begin{array}{l}\text { owns } \\
\text { latrine }\end{array}$ \\
\hline height-for-age, $z$-score & 0.068 & $0.031^{c}$ & 0.021 & 0.049 & $0.031^{c}$ & 0.021 & 0.029 & $0.031^{c}$ & 0.021 \\
\hline weight-for-age, $z$-score & -0.015 & $0.069^{d}$ & $0.112^{c}$ & 0.050 & $0.035^{c}$ & $0.058^{c}$ & 0.077 & 0.001 & 0.005 \\
\hline weight-for-height, $z$-score & -0.009 & 0.059 & 0.070 & 0.008 & 0.027 & 0.033 & 0.014 & -0.005 & -0.005 \\
\hline stunted prevalence, $\%$ & -2.99 & $-0.79^{c}$ & -0.49 & -2.42 & $-0.75^{c}$ & -0.47 & -1.88 & $-0.72^{c}$ & -0.46 \\
\hline underweight prevalence, \% & 0.25 & $-2.33^{d}$ & $-3.37^{d}$ & -1.35 & $-1.10^{d}$ & $-1.69^{c}$ & -2.28 & -0.10 & -0.49 \\
\hline wasting prevalence, $\%$ & -2.57 & -0.60 & 0.34 & -0.98 & -0.39 & 0.29 & 0.16 & -0.22 & 0.24 \\
\hline diarrhea prevalence, $\%$ & -3.13 & 0.47 & 0.54 & $-2.99^{b}$ & 0.50 & 0.58 & $-2.83^{c}$ & 0.52 & 0.61 \\
\hline $\log$ E. coli, MPN per $100 \mathrm{~mL}$ & 0.134 & $-0.078^{b}$ & $-0.191^{d}$ & -0.035 & -0.040 & $-0.095^{c}$ & -0.087 & -0.002 & 0.001 \\
\hline log total coliform, MPN per & 0.031 & -0.020 & -0.040 & -0.001 & -0.008 & $-0.025^{b}$ & -0.004 & 0.005 & -0.010 \\
\hline
\end{tabular}

${ }^{a}$ Effects are calculated for a $10 \%$ increase in community-level latrine coverage or a change from no household latrine to owning a household latrine. Note: Height-for-age, stunted prevalence, and diarrhea prevalence are calculated on the basis of a first-order regression with interaction term. All other outcomes are calculated on the basis of a second-order regression with interaction term. The full modeling results are provided in Table S3. ${ }^{b} p$ $<0.10{ }^{c} p<0.05{ }^{d}{ }^{d} p<0.01$. 
Table 3. Summary Results of Generalized Additive Models on Child Growth Outcomes and Household Stored Water Quality Relationships with Household Latrine Ownership and Percent Latrine Ownership among Neighbors ${ }^{a}$

\begin{tabular}{|c|c|c|c|c|c|c|c|}
\hline \multirow[b]{2}{*}{ outcome } & \multicolumn{2}{|c|}{ household latrine } & \multicolumn{2}{|c|}{$\begin{array}{l}\% \text { latrine coverage, households within } \\
\qquad 200 \mathrm{~m}\end{array}$} & \multirow[b]{2}{*}{ deviance explained, \% } & \multirow[b]{2}{*}{ adjusted- $R^{2}$} & \multirow[b]{2}{*}{$N$} \\
\hline & linear coefficient & $p$-value & estimated degrees of freedom & $p$-value & & & \\
\hline height-for-age, $z$-score & 0.039 & 0.389 & 2.250 & 0.001 & 10.8 & 0.104 & 5947 \\
\hline weight-for-age, $z$-score & 0.054 & 0.192 & 2.587 & 0.001 & 5.59 & 0.053 & 5953 \\
\hline weight-for-height, $z$-score & 0.005 & 0.898 & 2.278 & 0.017 & 6.76 & 0.065 & 5757 \\
\hline stunted prevalence, $\%$ & -0.024 & 0.067 & 1.711 & 0.007 & 5.40 & 0.050 & 5942 \\
\hline underweight prevalence, $\%$ & -0.020 & 0.138 & 2.453 & 0.000 & 3.45 & 0.032 & 5953 \\
\hline wasting prevalence, $\%$ & -0.004 & 0.730 & 1.000 & 0.493 & 5.91 & 0.057 & 5757 \\
\hline $\log$ E. coli, MPN per $100 \mathrm{~mL}$ & 0.004 & 0.963 & 2.251 & 0.055 & 14.1 & 0.126 & 773 \\
\hline log total coliform, MPN per $100 \mathrm{~mL}$ & -0.028 & 0.355 & 1.000 & 0.204 & 4.9 & 0.035 & 773 \\
\hline
\end{tabular}

For weight-for-age $z$-scores and underweight prevalence, second-order regression models including an interaction between community-level sanitation and household sanitation identified a statistically significant relationship with community latrine coverage; there was not a statistically significant association with weight-for-height $z$-scores or wasting (Tables S3 and S4). Statistically significant marginal effects estimated for an increase of $40 \%$ to $50 \%$ latrine coverage include a 0.031 $(p$-value $=0.040)$ increase in height-for-age $z$-score for households without a latrine, a $0.035(p$-value $=0.015)$ increase in weight-for-age $z$-score for households without a latrine, and a $0.058(p$-value $=0.043)$ increase in weight-for-age $z$-score for households with a latrine. Similarly, an increase from $40 \%$ to $50 \%$ latrine coverage was associated with a $0.75 \%$ ( $p$ value $=0.058)$ reduced prevalence of stunting for households and a $1.1 \%(p$-value $=0.002)$ reduced prevalence of underweight for households without a latrine and a $1.69 \%$ ( $p$ value $=0.018$ ) reduced prevalence of underweight for households with a latrine (Table 2). Individual household latrine ownership was not associated with child growth and did not have a significant moderating effect on latrine coverage for any of the health outcomes. Marginal effect estimates showed a marginally significant decrease in diarrhea prevalence for household latrine ownership of $2.9 \%(p$-value $=0.057)$ at $40 \%$ latrine coverage and a significant decrease of $2.8 \%$ ( $p$-value $=0.048$ ) at $60 \%$ latrine coverage, while increased community latrine coverage was not significantly associated with diarrhea prevalence (Table 2). The results of these regressions were not significantly different between male and female children for any health outcomes (Tables S6 and S7).

The generalized additive models confirmed community latrine coverage was significantly associated with child height and weight, while household latrine ownership was not significantly associated (wasting prevalence was not significantly associated with either household latrine ownership or community latrine coverage) (Table 3 ). The generalized additive model results for height-for-age suggest further increases in latrine coverage appear to no longer improve growth beyond approximately $75 \%$ (Figure 1); however, stunting exhibited a linear relationship and continued to decrease up to $100 \%$ latrine coverage (Figure 2). Clear nonlinear trends were observed in the generalized additive model results for underweight, weight-for-height, and weightfor-age child health measures (Figures 1 and 2). Above approximately $60 \%$ latrine coverage, improvements in weightfor-height and weight-for-age taper off and may decrease, although uncertainty in our estimates increase at higher levels of latrine coverage.

Water Quality. Household stored water samples had a geometric mean of $140 \mathrm{MPN}$ E. coli per $100 \mathrm{~mL}$ sample and 1642 MPN of total coliforms per $100 \mathrm{~mL}$ sample. Ninety-six percent of households had stored water covered at the time of sample collection; only $2 \%$ of households had treated the water by boiling or with chlorine. Forty-two percent of households collected drinking water from an improved source. Stored water quality summary statistics are provided in Table 1 .

Household stored water quality as indicated by E. coli concentrations was significantly associated with community sanitation coverage, although only at low-levels of community latrine coverage, while household latrine ownership was not associated with stored water quality (Table 2; regression results in Table S8). A higher percentage of households practicing open defecation in a $200 \mathrm{~m}$ radius had a statistically significant association with increased $E$. coli concentrations in the stored water $(p$-value $=0.021)$ and a marginally significant association with total coliform concentrations $(p$-value $=0.053)$. Open defecation at the household level was not associated with $E$. coli concentration $(p$-value $=0.089)$ (Table S9). The marginal effect calculated for an increase of $40 \%$ to $50 \%$ in community latrine coverage was a 0.095 ( $p$-value $=0.014) \log$ reduction in $E$. coli concentration for households with a latrine. Marginal effects at $20 \%$ coverage were larger with a 0.078 ( $p$-value $=$ $0.064) \log$ reduction of $E$. coli for households without a latrine and a 0.191 ( $p$-value $=0.006) \log$ reduction of $E$. coli for households with a latrine (Table 2).

Generalized additive model results showed a reduction in E. coli concentrations with increased community latrine coverage up to approximately $60 \%$ latrine coverage, followed by a leveling off and potential increase in concentration with increasing uncertainty at higher levels of community latrine coverage (Figure 1). Generalized additive models did not show any significant association between latrine ownership or latrine coverage and total coliform concentrations (Table 3).

No association was detected between sanitation coverage and drinking water source quality as indicated by E. coli concentrations (Table S10). There was a statistically significant association between total coliform concentrations and community latrine coverage $(p$-value $=0.022)$ as well as percent households practicing open defecation ( $p$-value $=$ 0.047).

Distance Robustness Check. A recent study analyzing the effect of community sanitation coverage in rural Ecuador used a $500 \mathrm{~m}$ radius definition of community. ${ }^{16}$ For all outcomes, we 

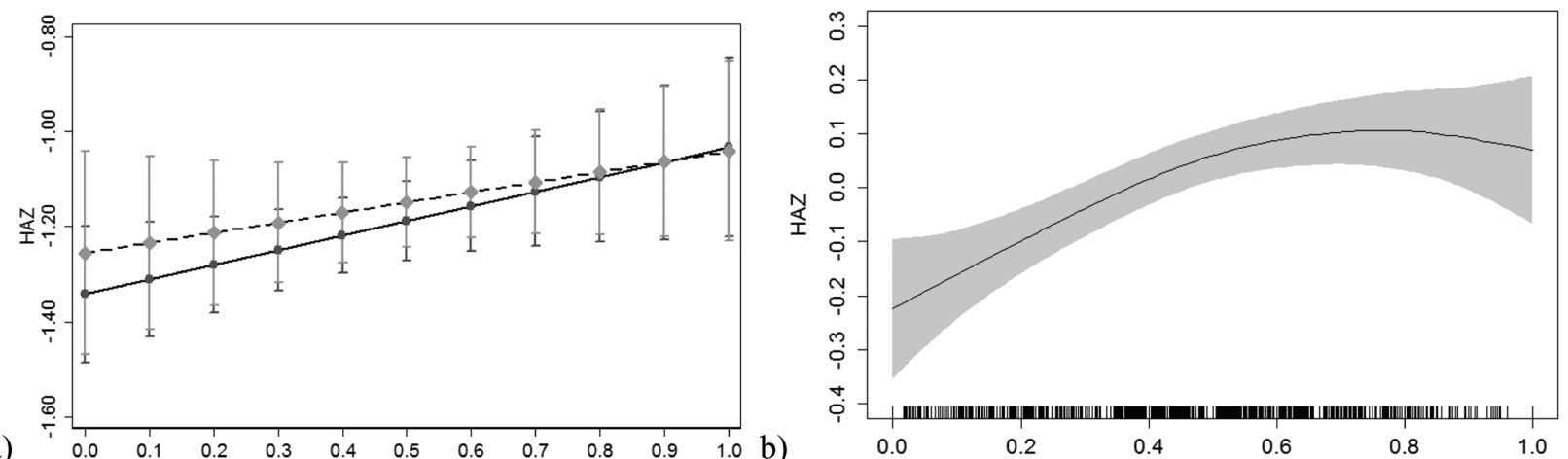

a)

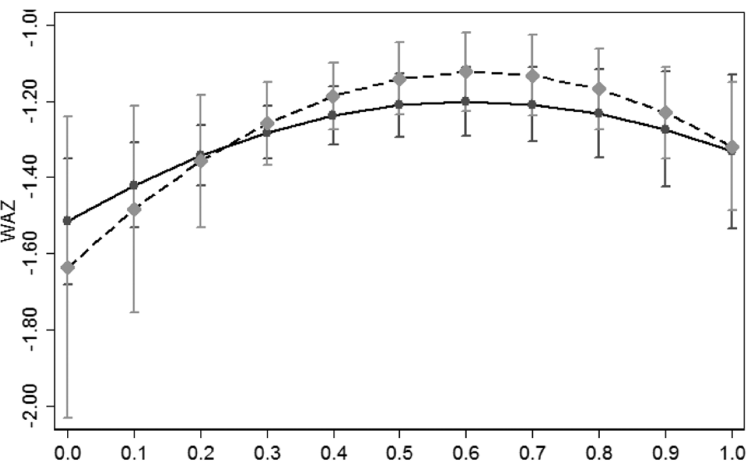

c)
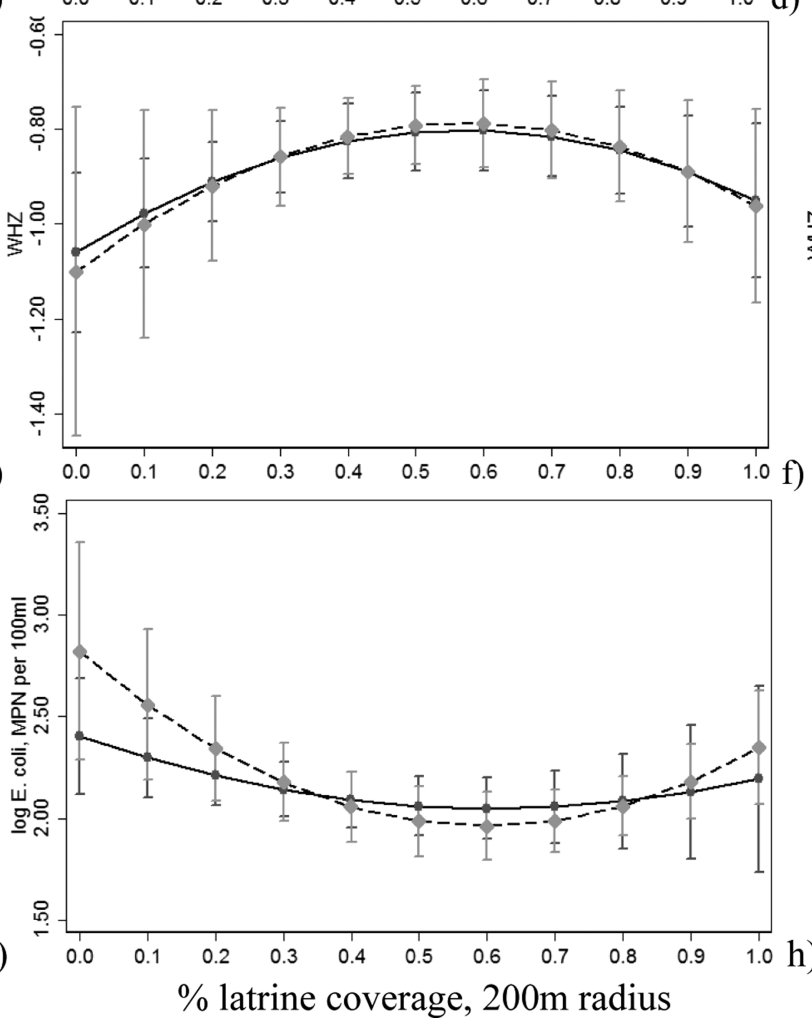

b)
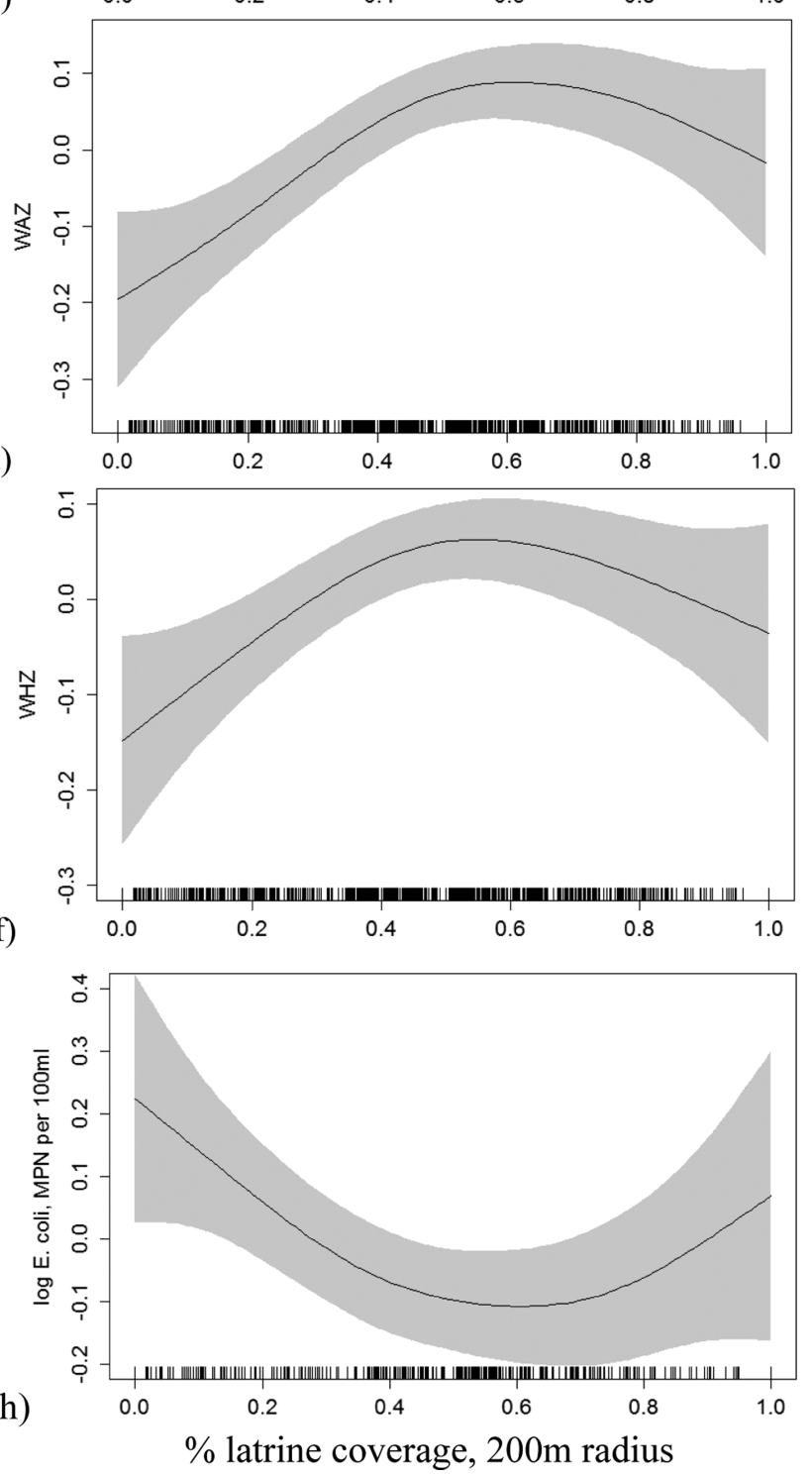

Figure 1. Estimated relationships between community latrine coverage and child growth and water quality. (a, c, e, g) (solid line, $\mathbf{\bullet})$ Household does not own a latrine; (dashed line, $\bullet$ ) Household owns a latrine. (a) Linear model results for height-for-age $z$-scores (HAZ). (c, e, g) Quadratic model results for weight-for-age $z$-scores (WAZ), weight-for-height $z$-scores (WHZ), and log E. coli concentrations, respectively. (b, d, f, h) Generalized additive modeling (GAM) results of the partial effect of sanitation coverage on the outcomes of HAZ, WAZ, WHZ and log E. coli concentrations, respectively-a 1.0 change on the $y$-axis represents a 1 unit change in the outcome, growth $z$-score or $\log E$. coli. Error bars and grey shaded areas represent $95 \%$ confidence intervals.

also completed the analysis using a radius of $500 \mathrm{~m}$ and $1 \mathrm{~km}$. Radii of less than $200 \mathrm{~m}$ were not useful in the study area due to low household density. These alternative models did not yield statistically different results; however, the $1 \mathrm{~km}$ radius did generate qualitatively different associations between weight-for- age and community latrine coverage and between weight-forheight and community latrine coverage. This difference was less apparent for binary outcomes of underweight and wasting (Tables S11 and S12). 

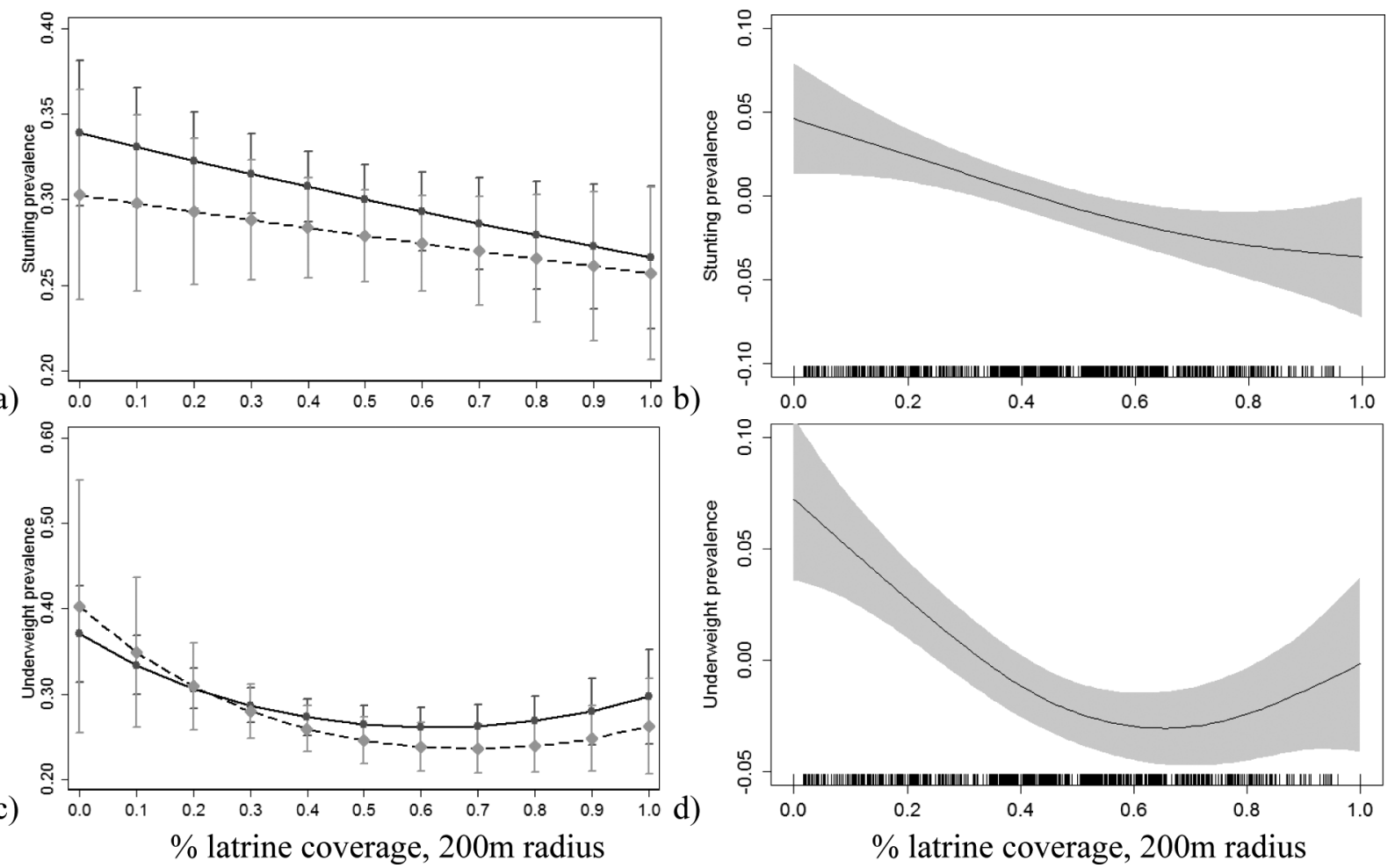

Figure 2. Relationship between community latrine coverage and child stunting and being underweight. (a, c) (solid line, $\mathbf{0}$ ) Household does not own a latrine; (dashed line, $\diamond$ ) Household owns a latrine. (a) Linear model results for stunting prevalence. (c) Quadratic model results for underweight prevalence. $(b, d)$ Generalized additive modeling (GAM) results of the partial effect around a mean of zero of sanitation coverage on the outcomes of stunting and underweight prevalence, respectively-a 0.1 decrease on the $y$-axis corresponds to a $10 \%$ reduction in prevalence. Error bars and grey shaded areas represent $95 \%$ confidence intervals.

\section{DISCUSSION}

Community latrine coverage was positively associated with child height and weight, while self-reported community open defecation rates were negatively associated with child growth. In contrast, individual household latrine ownership did not have statistically significant associations with child health in our study population. We found no significant difference in results when an interaction term between community sanitation coverage and household latrine ownership was included, indicating the association between child health and community latrine coverage did not significantly differ depending on individual household latrine ownership. Notably, the marginal effects of increasing community latrine coverage were greater for households that did not own a latrine, indicating the importance of accounting for this interaction. Our findings are consistent with a recent study in rural Ecuador that identified reduced stunting prevalence in communities with overall improved sanitation access yet found no impact of individual household improved latrine ownership on stunting. Notably, the Ecuador study site had very low open defecation rates; our study is able to contribute new evidence for the health benefits of communities transitioning from prevalent open defecation to basic latrine usage.

We observed a linear relationship between community latrine coverage and stunted growth, indicating that marginal increases in community latrine coverage up to $100 \%$ may offer additional improvements in child height in rural Mali (i.e., we did not identify a community coverage threshold after which child linear growth benefits significantly tapered off). However, we observed a nonlinear relationship between sanitation coverage and a child being underweight, suggesting effects on child weight may level off with improvements in sanitation coverage beyond $60 \%$. The generalized additive modeling results supported a linear relationship for child height and a nonlinear relationship for child weight. High variability in the data presents some uncertainty at high levels of coverage for both stunting and underweight prevalence. The Ecuador study reported a rapid decrease in stunting prevalence going from 0 to $30 \%$ improved sanitation coverage, with reductions mostly ceasing beyond $30 \%$ improved sanitation coverage. ${ }^{16}$ On the contrary, in Indian villages, almost no reductions in diarrhea prevalence were found below 30\% improved latrine coverage, while the majority of health gains were after $75 \%$ improved latrine coverage was achieved. ${ }^{19}$ Potential explanations for these differing results include variations in climate, population density, and seasonality of data collection ${ }^{29,30}$ and the examination of improved latrine coverage versus the transition from open defecation to basic sanitation.

Household stored water quality was found to improve with increased community latrine coverage and less open defecation near the household. This finding supports the hypothesis that water contamination is one pathway through which sanitation coverage affects child growth. Contamination of drinking water stored in the home has been shown in other studies to be a risk factor for child diarrhea regardless of source water quality; $^{25,26,31,32}$ this contamination risk could increase if the nearby environment has higher fecal contamination from persistent open defecation within a community. For example, increased hand fecal contamination has previously been found to be associated with unimproved sanitation access as well as high levels of fecal contamination in stored drinking water. ${ }^{33}$ We did not detect a significant association between source water quality 
and sanitation coverage in our study area; one potential explanation is the groundwater table in Mali might be too deep to be affected by open defecation or fecal pollution from pit latrines. $^{34}$

This study has some important limitations. The crosssectional data set limits the identification of causal relationships. Stunting has been found to occur largely from prebirth to two years of age; ${ }^{35}$ however, we only had data on concurrent sanitation conditions and health (previous exposure to sanitation infrastructure was not known). Confounding variables, such as wealth or education, could have contributed to overestimation of the reported associations. However, controlling for wealth and literacy at the household level and at the community level (defined either by village boundaries or by a 200 m radius) did not significantly alter our results (Tables S3 and S13). Community-level wealth and education averages were used by Fuller et al. ${ }^{16}$ in Ecuador, yet village- or community-level variables have been neglected in other sanitation studies. ${ }^{18,36}$ Additionally, we were unable to control for child nutritional intake, which may alter growth outcomes and diarrhea prevalence. We also were not able to account for distance of open defecation location from the index household. Prior research has also shown that open defecation or unimproved sanitation may present greater risk to child growth in densely populated areas. ${ }^{37}$ Our study was completed in rural Mali and therefore does not address the importance of latrine coverage in densely populated urban settings. When we conducted two separate analyses that excluded the $10 \%$ most densely populated and the $10 \%$ least densely populated, our results did not significantly change (data not shown). The relatively low population density in rural Mali may explain some of the variation between our findings and those from rural India, where population density was higher. ${ }^{17}$

The post-2015 sustainable development goals aim for a complete end to open defecation and universal access to private improved sanitation infrastructure. We found that benefits to child health may result from increases in basic sanitation coverage up to $100 \%$. Previous studies identified lower thresholds of sanitation coverage, yet they either focused on diarrhea prevalence as the primary health indicator or examined transitions from unimproved sanitation to improved sanitation infrastructure. While our findings may not apply to settings with higher population densities, they indicate that access to basic sanitation is the first step to protect child health in a rural setting. The positive sanitation externalities identified in this study justify efforts to increase access to basic sanitation infrastructure, even if open defecation cannot be fully eliminated in the immediate future.

\section{ASSOCIATED CONTENT}

\section{S Supporting Information}

The Supporting Information is available free of charge on the ACS Publications website at DOI: 10.1021/acs.est.7b00178.

Additional results regarding open defecation practices in the community and relationships to latrine coverage, child health outcomes, and water quality; sensitivity analyses on child gender and community-size specifications of $500 \mathrm{~m}$ and $1 \mathrm{~km}$; localized polynomials for growth and water quality as well as generalized additive models for binary outcomes; regression results for firstorder and second-order linear regressions with the full list of covariates; a comparison of regression results between village-level wealth and literacy and communitylevel (200m radius) wealth and literacy; results and methods of the limited source water quality analysis within the study site (PDF)

\section{AUTHOR INFORMATION}

\section{Corresponding Author}

*E-mail: amyjanel@gmail.com.

ORCID

Amy Pickering: 0000-0001-6193-2221

Notes

The authors declare no competing financial interest.

\section{ACKNOWLEDGMENTS}

Funding was provided by The Bill \& Melinda Gates Foundation. Harris was supported by a Science to Achieve Results (STAR) Fellowship from the US EPA and the Landreth I Fellowship in the Emmett Interdisciplinary Program in Environment and Resources. We would like to thank Diana Pinto for contributing to the design of the survey instruments; Maria Adelaida Lopera, Moussa Cissoko, and Moussa Coulibaly for assistance with data collection; Carolina Lopez for data processing. We thank Habiba Djebbari for providing useful feedback on the manuscript draft.

\section{REFERENCES}

(1) United Nations. Transforming our world: the 2030 Agenda for Sustainable Development; United Nations: New York, 2015.

(2) WHO; UNICEF. 25 Years Progress on Sanitation and Drinking Water: 2015 Update and MDG Assessment; WHO Press: Geneva, 2015.

(3) Prüss, A.; Kay, D.; Fewtrell, L.; Bartram, J. Estimating the burden of disease from water, sanitation, and hygiene at a global level. Environ. Health Perspect. 2002, 110 (5), 537-542.

(4) Prüss-Ustün, A.; Bartram, J.; Clasen, T.; Colford, J. M.; Cumming, O.; Curtis, V.; Bonjour, S.; Dangour, A. D.; De France, J.; Fewtrell, L.; et al. Burden of disease from inadequate water, sanitation and hygiene in low- and middle-income settings: A retrospective analysis of data from 145 countries. Trop. Med. Int. Health 2014, 19 (8), 894-905.

(5) Humphrey, J. H. Child undernutrition, tropical enteropathy, toilets, and handwashing. Lancet 2009, 374 (9694), 1032-1035.

(6) Guerrant, R. L.; DeBoer, M. D.; Moore, S. R.; Scharf, R. J.; Lima, A. M. The impoverished gut-a triple burden of diarrhoea, stunting and chronic disease. Nat. Rev. Gastroenterol. Hepatol. 2013, 10 (4), 220229.

(7) Lin, A.; Arnold, B. F.; Afreen, S.; Goto, R.; Huda, T. M. N.; Haque, R.; Raqib, R.; Unicomb, L.; Ahmed, T.; Colford, J. M.; et al. Household environmental conditions are associated with enteropathy and impaired growth in rural bangladesh. Am. J. Trop. Med. Hyg. 2013, 89 (1), 130-137.

(8) Spears, D.; Ghosh, A.; Cumming, O. Open defecation and childhood stunting in India: an ecological analysis of new data from 112 districts. PLoS One 2013, 8 (9), e73784.

(9) Pickering, A. J.; Djebbari, H.; Lopez, C.; Coulibaly, M.; Alzua, M. L. Effect of a community-led sanitation intervention on child diarrhoea and child growth in rural Mali: a cluster-randomised controlled trial. Lancet Glob. Heal. 2015, 3 (11), e701-e711.

(10) Clasen, T.; Boisson, S.; Routray, P.; Torondel, B.; Bell, M.; Cumming, O.; Ensink, J.; Freeman, M.; Jenkins, M.; Odagiri, M.; et al. Effectiveness of a rural sanitation programme on diarrhoea, soiltransmitted helminth infection, and child malnutrition in Odisha, India: a cluster-randomised trial. Lancet Glob. Heal. 2014, 2 (11), e645-e653. 
(11) Cairncross, S.; Shordt, K.; Zacharia, S.; Govindan, B. K. What causes sustainable changes in hygiene behaviour? A cross-sectional study from Kerala, India. Soc. Sci. Med. 2005, 61 (10), 2212-2220.

(12) Schmidt, W. P. Seven trials, seven question marks. Lancet Glob. Heal. 2015, 3 (11), e659-e660.

(13) VanDerslice, J.; Briscoe, J. All coliforms are not created equal: A comparison of the effects of water source and in-house water contamination and infantile diarrheal disease. Water Resour. Res. 1993, 29 (7), 1983-1995.

(14) Trevett, A. F.; Carter, R. C.; Tyrrel, S. F. The importance of domestic water quality management in the context of faecal-oral disease transmission. J. Water Health 2005, 3 (3), 259-270.

(15) Root, G. P. M. Sanitation, Community Environments, and Childhood Diarrhea in Rural Zimbabwe. J. Heal. Popul. Nutr. 2001, 19 (2), 73-82.

(16) Fuller, J. A.; Villamor, E.; Cevallos, W.; Trostle, J.; Eisenberg, J. N. S. I get height with a little help from my friends: Herd protection from sanitation on child growth in rural Ecuador. Int. J. Epidemiol. 2016, 45 (2), 460-469.

(17) Andres, L. A.; Briceno, B.; Chase, C.; Echenique, J. Sanitation and Externalities: Evidence from Early Childhood Health in Rural India; Policy Research Working Paper No. 6737; The World Bank: Washington, DC, 2014.

(18) Perchoux, C.; Chaix, B.; Cummins, S.; Kestens, Y. Conceptualization and measurement of environmental exposure in epidemiology: Accounting for activity space related to daily mobility. Heal. Place 2013, 21, 86-93.

(19) Spears, D. How Much International Variation in Child Height Can Sanitation Explain? World Bank Policy Research Work. Pap. 2013, 6351 (February), 1-53.

(20) Geruso, M.; Spears, D. Neighborhood Sanitation and Infant Mortality. National Bureau of Economic Research Work. Pap. 2015, 21184 (May), 1-55.

(21) Verhougstraete, M. P.; Martin, S. L.; Kendall, A. D.; Hyndman, D. W.; Rose, J. B. Linking fecal bacteria in rivers to landscape, geochemical, and hydrologic factors and sources at the basin scale. Proc. Natl. Acad. Sci. U. S. A. 2015, 112 (33), 10419-10424.

(22) Wright, J. A.; Cronin, A.; Pedley, S.; Gundry, S. W.; et al. A spatial analysis of pit latrine density and groundwater source contamination. Environ. Monit. Assess. 2013, 185 (5), 4261-4272.

(23) Nsubuga, F. B.; Kansiime, F.; Okot-Okumu, J. Pollution of protected springs in relation to high and low density settlements in Kampala-Uganda. Phys. Chem. Earth, Parts A/B/C 2004, 29 (1518), 1153-1159.

(24) Adekunle, I. M.; Adetunji, M. T.; Gbadebo, A. M.; Banjoko, O. P. Assessment of groundwater quality in a typical rural settlement in southwest Nigeria. Int. J. Environ. Res. Public Health 2007, 4 (4), 307318.

(25) Wright, J.; Gundry, S.; Conroy, R. Household drinking water in developing countries: A systematic review of microbiological contamination between source and point-of-use. Trop. Med. Int. Health 2004, 9 (1), 106-117.

(26) Schriewer, A.; Odagiri, M.; Wuertz, S.; Misra, P. R.; Panigrahi, P.; Clasen, T.; Jenkins, M. W. Human and Animal Fecal Contamination of Community Water Sources, Stored Drinking Water and Hands in Rural India Measured with Validated Microbial Source Tracking Assays. Am. J. Trop. Med. Hyg. 2015, 93 (3), 509516.

(27) UN-Water. Metadata on Suggested Indicators for Global Monitoring of the Sustainable Development Goal 6 on Water and Sanitation; United Nations: New York, 2015.

(28) Vazquez-Prokopec, G. M.; Bisanzio, D.; Stoddard, S. T.; PazSoldan, V.; Morrison, A. C.; Elder, J. P.; Ramirez-Paredes, J.; Halsey, E. S.; Kochel, T. J.; Scott, T. W.; et al. Using GPS Technology to Quantify Human Mobility, Dynamic Contacts and Infectious Disease Dynamics in a Resource-Poor Urban Environment. PLoS One 2013, 8 (4), e58802.

(29) Carlton, E. J.; Eisenberg, J. N. S.; Goldstick, J.; Cevallos, W.; Trostle, J.; Levy, K. Heavy rainfall events and diarrhea incidence: The role of social and environmental factors. Am. J. Epidemiol. 2014, 179 (3), 344-352.

(30) Bhavnani, D.; Goldstick, J. E.; Cevallos, W.; Trueba, G.; Eisenberg, J. N. S. Impact of rainfall on diarrheal disease risk associated with unimproved water and sanitation. Am. J. Trop. Med. Hyg. 2014, 90 (4), 705-711.

(31) Clasen, T. F.; Bastable, A. Faecal contamination of drinking water during collection and household storage: The need to extend protection to the point of use. J. Water Health 2003, 1 (3), 109-115.

(32) Harris, A. R.; Boehm, A. B.; Davis, J. Mechanisms of post-supply contamination of drinking water in Bagamoyo, Tanzania. J. Water Health 2013, 11 (3), 543-554.

(33) Pickering, A. J.; Davis, J.; Walters, S. P.; Horak, H. M.; Keymer, D. P.; Mushi, D.; Strickfaden, R.; Chynoweth, J. S.; Liu, J.; Blum, A.; et al. Hands, water, and health: Fecal contamination in Tanzanian communities with improved, non-networked water supplies. Environ. Sci. Technol. 2010, 44 (9), 3267-3272.

(34) British Geological Survey; WaterAid. Groundwater Quality: Mali; British Geological Survey: Keyworth,2002.

(35) Victora, C. G.; de Onis, M.; Hallal, P. C.; Blössner, M.; Shrimpton, R. Worldwide timing of growth faltering: revisiting implications for interventions. Pediatrics 2010, 125 (3), e473-e480.

(36) Fuller, J. A.; Eisenberg, J. N. S. Herd protection from drinking water, sanitation, and hygiene interventions. Am. J. Trop. Med. Hyg. 2016, 95 (5), 1201-1210.

(37) Hathi, P.; Haque, S.; Pant, L.; Coffey, D.; Spears, D. Place and Child Health: The Interaction of Population Density and Sanitation in Developing Countries; 7124; World Bank Group: Washington, DC, 2014. 